OPEN ACCESS

Edited by:

Mandy Laube

Leipzig University, Germany

Reviewed by:

Carla Bellinghausen,

Goethe University Frankfurt, Germany

Sompong Vongpunsawad,

Chulalongkorn University, Thailand

*Correspondence:

Yuqing Wang

wang_yu_qing@126.com

†These authors have contributed equally to this work

Specialty section:

This article was submitted to

Pediatric Pulmonology,

a section of the journal

Frontiers in Pediatrics

Received: 26 October 2020

Accepted: 29 June 2021

Published: 12 August 2021

Citation:

Chen S, Wang Y, Li A, Jiang W, Xu Q, Wu M, Chen Z, Hao C, Shao $X$ and Xu J (2021) Etiologies of Hospitalized Acute Bronchiolitis in Children 2 Years of Age and Younger: A 3 Years' Study

During a Pertussis Epidemic.

Front. Pediatr. 9:621381

doi: 10.3389/fped.2021.621381

\section{Etiologies of Hospitalized Acute Bronchiolitis in Children 2 Years of Age and Younger: A 3 Years' Study During a Pertussis Epidemic}

\author{
Sainan Chen ${ }^{1 \dagger}$, Yuqing Wang ${ }^{1 *}$, Anrong $\mathrm{Li}^{1}$, Wujun Jiang ${ }^{1+}$, Qiuyan $\mathrm{Xu}^{2}$, Min $\mathrm{Wu}^{1}$, \\ Zhengrong Chen ${ }^{1}$, Chuangli Hao ${ }^{1}$, Xunjun Shao ${ }^{1}$ and Jun $\mathrm{Xu}^{1}$
}

${ }^{1}$ Department of Respiratory Medicine, Children's Hospital of Soochow University, Suzhou, China, ${ }^{2}$ Department of Pediatrics, Affiliated Suzhou Science and Technology Town Hospital of Nanjing Medical University, Suzhou, China

Objective: In recent years, the incidence of Bordetella pertussis infection in infants and young children has been increasing. Multiple studies have suggested that $B$. pertussis may be one of the pathogens of bronchiolitis in infants and young children. However, the prevalence and clinic characteristic of $B$. pertussis in bronchiolitis is controversial. This prospective descriptive study evaluated the prevalence and clinical manifestations of infants and young children hospitalized for bronchiolitis with B. pertussis.

Methods: Children hospitalized with bronchiolitis were eligible for a prospective study for 36 months from January 1, 2017, to December 31, 2019. Besides B. pertussis, 10 common respiratory viruses and Mycoplasma pneumoniae (MP) were confirmed by laboratory tests. Medical records of patients were reviewed for demographic, clinical characteristics, and laboratory examination.

Results: A total of 1,092 patients with bronchiolitis were admitted. B. pertussis was detected in 78/1,092 (7.1\%) patients. Of the 78 patients with B. pertussis bronchiolitis, coinfections occurred in $45(57.7 \%)$ patients, most frequently with human rhinovirus (28/78, 35.9\%), followed by MP (9/78, 11.4\%), and human bocavirus $(6 / 78,7.7 \%)$. The peak incidence of $B$. pertussis infection was in May. A high leukocyte count could help distinguish $B$. pertussis-associated acute bronchiolitis from other acute bronchiolitis etiologies. After excluding coinfections, children with B. pertussis-only bronchiolitis exhibited a milder clinical presentation than those with RSV-only infection; also, children with MP-only and other pathogen infections revealed similar severity. The morbidity of $B$. pertussis was common $(31 / 78,39.7 \%)$ in infants with bronchiolitis under 3 months.

Conclusion: In summary, B. pertussis is one of the pathogens in children with bronchiolitis, and coinfection of $B$. pertussis with other viruses is common in bronchiolitis. B. pertussis should be considered when patients hospitalized with bronchiolitis present a longer course and have an elevated leukocyte count. Patients with $B$. pertussis-associated bronchiolitis present a milder clinical presentation.

Keywords: Bordetella pertussis, bronchiolitis, coinfection, immunization, disease progression, high leukocyte count 


\section{INTRODUCTION}

Pertussis, caused by the bacterium Bordetella pertussis, is a highly contagious respiratory disease and one of the leading causes of death from infectious diseases in children. B. pertussis, a Gram-negative bacterium that was first described by Bordet and Gengou in 1906 (1), has recently reemerged as a major public health threat. The World Health Organization reported 141,074 confirmed pertussis cases worldwide in 2018 (2). Approximately 160,700 deaths were reported worldwide in 2014 from pertussis in children $<5$ years of age ( 3 ).

Bronchiolitis is the most common acute respiratory disease in infants and young children, and one of the most common causes of hospital admission $(4,5)$. A total of $40-80 \%$ of infection is caused by respiratory syncytial virus (RSV), followed by human rhinovirus (HRV), adenovirus (ADV), parainfluenza virus, human bocavirus (hBoV), and human metapneumovirus (hMPV) $(6,7)$.

In recent years, several studies suggested that $B$. pertussis is a possible pathogen causing bronchiolitis in infants and young children hospitalized for lower respiratory tract infections (810). However, studies reporting the prevalence and clinical characteristics of $B$. pertussis bronchiolitis are rare. This study aimed to assess the epidemiological features and clinical characteristics of $B$. pertussis infection and evaluate its impact on infants and young children hospitalized with acute bronchiolitis.

\section{MATERIALS AND METHODS}

\section{Patients and Definitions}

This prospective descriptive study was conducted on children presenting with acute bronchiolitis who were admitted to the Department of Respiratory Medicine in the Children's Hospital of Soochow University between January 1, 2017, and December 31, 2019. Acute bronchiolitis was characterized by age $\leq 2$, cough, tachypnea, retraction, and expiratory wheezes, often accompanied by rales (11). B. pertussis was confirmed by polymerase chain reaction (PCR) assays (12). Patients requiring oxygen supply were considered with severe conditions. The exclusion criteria were as follows: (1) patients with incomplete clinical data; (2) patients with bronchopulmonary dysplasia, heredity metabolic diseases, neurological disorders, congenital heart disease, and immunodeficiency; and (3) patients with evidence suggesting that wheezing was caused by tuberculosis and non-infectious factors such as bronchial foreign bodies.

The study was approved by the ethics committees of Children's Hospital Soochow University (Approval No. 2016026). Informed consent was obtained from the parents of all children enrolled in this study.

\section{Determination of Vaccination Status}

Vaccination history was obtained by querying the "Suzhou Children's Vaccination Inquiry and Evaluation Platform.” A diphtheria, tetanus, and acellular pertussis combination vaccine was administered as a primary series at 3, 4, and 5 months, followed by a booster dose at 24 months in China. The
TABLE 1 | Gene primer sequence and product length detected by real-time PCR.

\begin{tabular}{llc}
\hline Gene name & Primer sequence products & Length \\
\hline IS481 & 5'GATTCAATAGGTTGTATGCATGGTT3' & 145 \\
& 5'TGGACCATTTCGAGTCGACG3' & \\
PtXA-pr & 5'CCAACGCGCATGCGTGCAGATTCGTC3' & 191 \\
& 5'CCCTCTGCGTTTGATGGTGCCTATITA3' & \\
\hline
\end{tabular}

vaccination status was regarded as ever-vaccinated if one to three doses were received.

\section{Data Collection}

Data regarding demographic, clinical, and laboratory characteristics were documented. Demographic and clinical characteristics included age, gender, length of hospital stay, and requirement of supplemental oxygen. Laboratory specimens were obtained including blood and nasopharyngeal aspirates (NPAs). NPAs were obtained during the first $24 \mathrm{~h}$ of hospitalization, using a sterile plastic catheter briefly inserted into the lower pharynx via the nasal cavity. The blood samples were taken immediately after hospitalization. The laboratory data of leukocyte count, percentages of lymphocytes and neutrophils, and detection of common viruses were collected.

\section{PCR Detection of $B$. pertussis}

B. pertussis DNA was detected in NPAs by real-time PCR assays. The primer sequence was synthesized by Shanghai Sangon Biotech Company. The pertussis PtxA-pr and IS481 gene sequences were used as specific primers (Table 1). The RTPCR assay result was considered negative if the cycle threshold (CT) was $\geq 40$. Specimens that tested positive by PCR for both insertion sequence IS481 (CT $<40)$ and ptxS1 $(\mathrm{CT}<40)$ were considered positive for $B$. pertussis. If a specimen was PtxApr target negative with an IS481 assay CT $<35$, it was also considered positive for $B$. pertussis.

\section{Respiratory Pathogens}

Direct immunofluorescence was used to detect RSV; ADV; influenza virus A (IV-A) and B (IV-B); and parainfluenza virus 1 (PIV I), 2 (PIV II), and 3 (PIV III) using a $\mathrm{D}^{3}$ Ultra Respiratory Virus Screening and LD Kit (Diagnostic Hybrids, Athens, OH, USA). A positive result was defined as over five inclusion bodies analyzed under a fluorescence microscope. Mycoplasma pneumoniae (MP), HRV, HMPV, and HBoV were detected by a PCR (nucleic acid amplification fluorescent reagent kit, Ann Gene Co., Guangdong, China) according to the manufacturer's instructions.

\section{Statistical Analyses}

Statistical analyses were conducted using SPSS 26.0 (IBM, SPSS, Chicago, IL, USA).

Data were shown as mean \pm standard deviation and median and interquartile range. Quantitative variables among the three age groups were compared using one-way analysis of variance or the Kruskal-Wallis test when appropriate. Frequency 


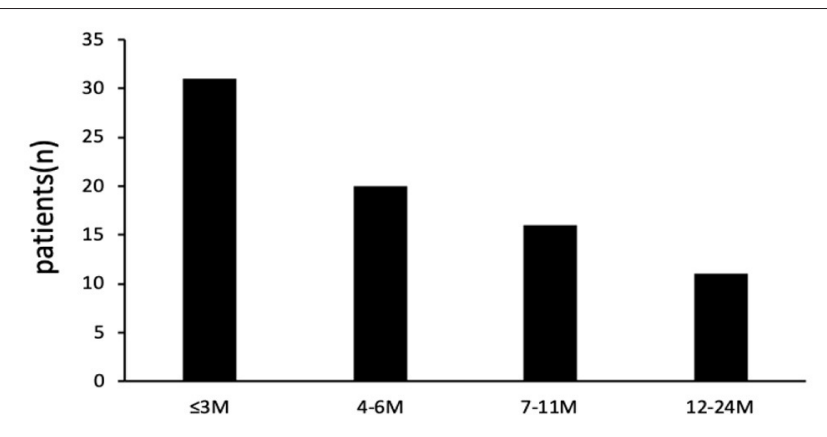

FIGURE 1 | Distribution of $B$. pertussis infection in infants and young children stratified by age.

distribution was compared by the chi-square test. A $p$ value $<0.05$ was considered as a significant difference.

\section{RESULTS}

\section{Demographic Characteristics}

Of the total 1,092 patients admitted for bronchiolitis, one or more respiratory pathogens including virus and MP were detected in 1,057 of 1,092 patients (a positive rate of $96.8 \%$ ) and B. pertussis was identified in 78 patients $(7.1 \%$, based on positive results by PCR). Of the 78 cases of bronchiolitis with $B$. pertussis infection, $47(60.3 \%)$ were male and $31(39.7 \%)$ were female. The male-tofemale ratio was 1.52:1. The median age was $6.45 \pm 4.94$ months. The age distribution of patients is shown in Figure 1; 31 (39.7\%) patients were aged $\leq 3$ months, $20(25.6 \%)$ patients were aged $4-$ 6 months, $16(20.5 \%)$ patients were aged 7-11 months, and 11 (14.1\%) patients were aged $\geq 12$ months.

\section{Seasonality of $B$. pertussis Infection}

The monthly distribution of $B$. pertussis infection is shown in Figures 2A,B. Bronchiolitis could occur throughout the year, and the peak incidence was in winter. The most common pathogen of bronchiolitis was RSV (534/1092, 48.9\%), and the peak incidence was in December. MP was detected in 159/1,092 (14.6\%) children with bronchiolitis, and the peak incidence was in September. Differing from the above two pathogens, the peak incidence of B. pertussis infection was in May, with a total of $10(19.2 \%, 10 / 52)$ patients reported, and no patients were infected in October and December.

\section{Coinfection Status}

Overall, one or more respiratory pathogens including virus and MP were detected in 1,057 of 1,092 patients. The most commonly detected pathogens in patients with bronchiolitis were as follows: RSV (48.9\%), HRV (25.9\%), HMPV (13.0\%), MP (14.6\%), HBoV (12.1\%), B. pertussis (7.1\%), PIV III (7.0\%), ADV (1.1\%), and PIV I (1.1\%).

Of the $78 \mathrm{~B}$. pertussis-infected patients, $B$. pertussis was the sole pathogen detected in $33(42.3 \%)$ patients. The remaining 45 patients $(57.7 \%)$ were coinfected with other respiratory pathogens, most frequently with $\operatorname{HRV}(n=28,35.9 \%)$, followed by $\operatorname{MP}(n=9,11.4 \%), \operatorname{HBoV}(n=6,7.7 \%), \operatorname{PIV} \operatorname{III}(n=4,5.1 \%)$, $\operatorname{RSV}(n=3,3.9 \%), \operatorname{IV}-\mathrm{A}(n=3,3.9 \%)$, and HMPV $(n=2,2.6 \%)$ (Figure 3).

\section{Clinical Features of B. pertussis-Only Infection Compared With Infections With Other Pathogens}

In the present study, 33 patients with $B$. pertussis-only infection, 438 patients with RSV-only infection, 87 patients with MP-only infection, and 534 patients infected with other pathogens were analyzed. In unadjusted comparisons, children with $B$. pertussisonly infection were similar to children with RSV-only infection in age, but the number of children with age $\leq 3$ months who were only infected with pertussis was less than that of children with RSV-only infection (Table 2). Children with B. pertussisonly infection were significantly more likely to have vomiting (36.4\%), cyanosis $(12.1 \%)$, leukocyte count $>15 \times 10^{9}(57.6 \%)$, longer duration of symptoms before admission (media day, 14.0), and longer hospital stay (media day, 9.0) compared with those with RSV-only infection $(17.8 \%, 2.7 \%, 7.5 \%$, media 5.0 , and 8.0 days, respectively; $p<0.05$ for all comparisons). Patients with $B$. pertussis-only infection requiring supplement oxygen were fewer than patients with RSV-only infection (6.1 vs. $34.9 \%$; $p<0.05$ ).

In unadjusted comparisons, among 120 patients with $B$. pertussis and MP infections excluding co-detection with other pathogen types, children with $B$. pertussis-only infection were younger than children with MP-only infection (median 3.9 vs. 5.8 months, respectively) (Table 2). Children with $B$. pertussis-only infection were significantly more likely to have dyspnea $(6.1 \%)$, rhinorrhea (15\%), vomiting (15\%), cyanosis (4\%), and leukocyte count $>15 \times 10^{9} / \mathrm{L}(57.6 \%)$ compared with those with MP-only infection $(0.0,24.1,10.3,0.0$, and $6.9 \%$, respectively; $p<0.05$ for all comparisons). Children with $B$. pertussis-only infection had a higher number of leukocyte and higher percentage of lymphocyte compared with children with MP-only infection. Children with B. pertussis-only infection had a longer duration of hospital stay (median 9.0 days) than those with MP-only infection (median 8.0 days); however, no significant difference was observed in the duration of symptoms before admission.

\section{B. pertussis Infection and Results of Laboratory Examination in Different Age Groups}

B. pertussis-positive patients were divided into three age groups to assess the difference among different age groups (Table 3). A total of 31 patients aged $\leq 3$ months, 36 patients aged 4-11 months, and 11 patients aged $\geq 12$ months were analyzed. Patients aged $\leq 3$ months had a longer duration of hospital stay than others $(p<0.05)$. The common clinic characteristics among the 78 confirmed patients were paroxysmal cough $92.3 \%$ (72/78), whoops $15.5 \%$ (12/78), post-tussive vomiting $38.5 \%(30 / 78)$, and cyanosis $12.8 \%$ (10/78). Patients with cyanosis aged $\leq 3$ months were more compared with older ones $(p<0.05)$; the others exhibited no difference among three age groups $(p>0.05)$. Patients aged $\leq 3$ months requiring supplemental oxygen were more compared 


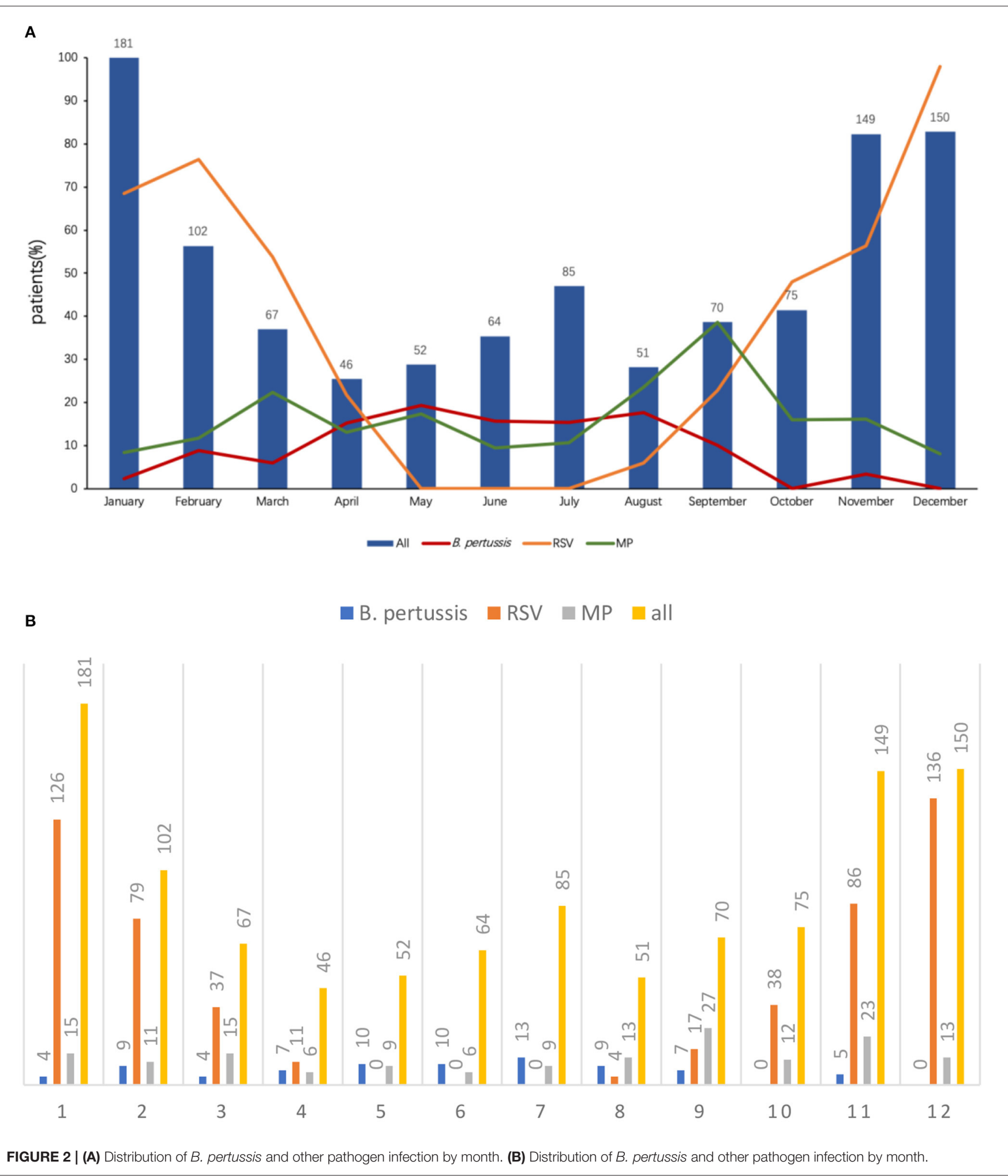

with older ones $(p<0.05)$. The gender ratio exhibited no significant difference among the three groups $(p<0.05)$. Coinfection among the three age groups was also compared, which showed no difference $(p>0.05)$. Although, patients aged $\geq 12$ months had a higher number of leukocytes, and higher percentages of neutrophils and lymphocytes, no 


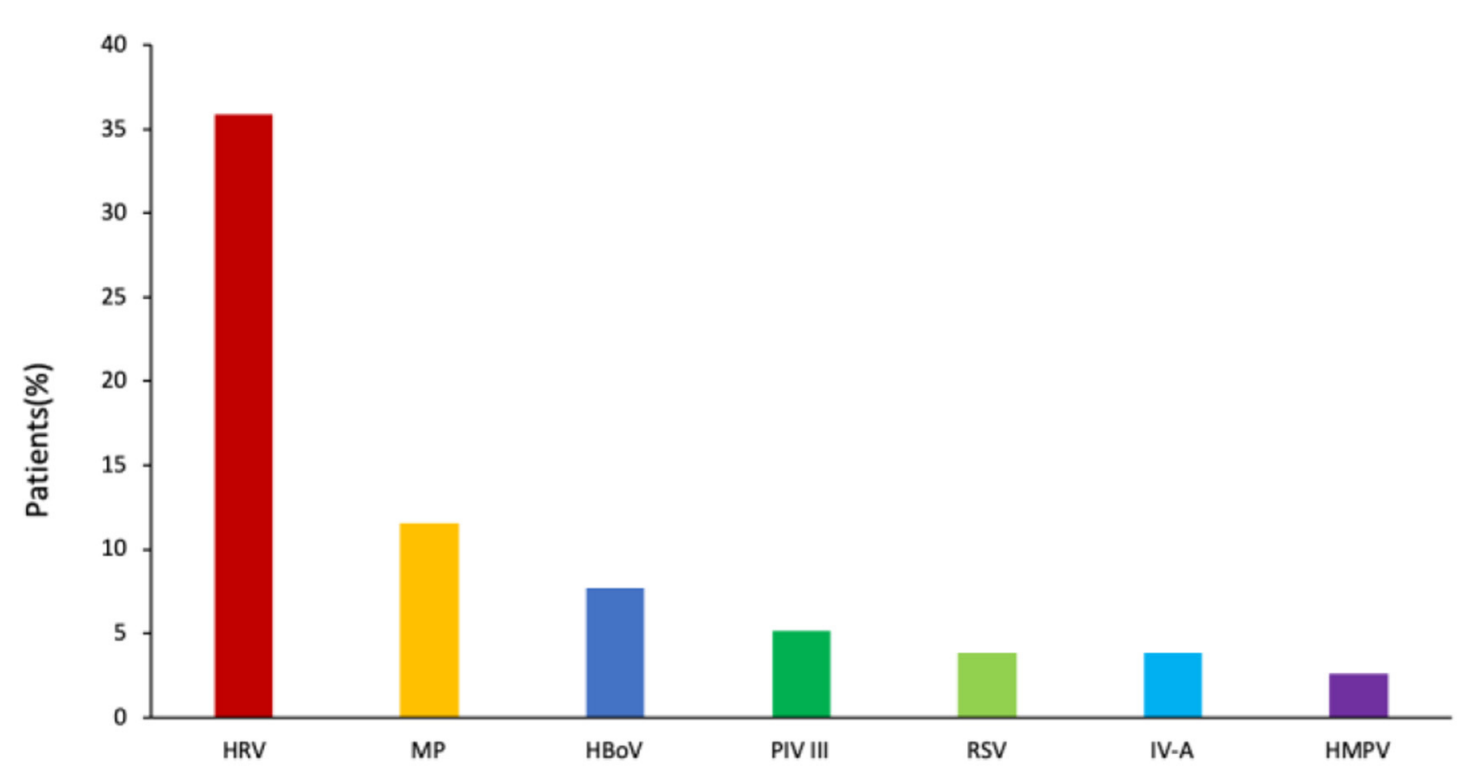

FIGURE 3 | Distribution of pathogen coinfections associated with B. pertussis infection.

significant difference was observed among the three groups ( $p$ $>0.05)$.

\section{Clinical Features of B. pertussis-Only Infection Compared With Coinfection}

Patients with $B$. pertussis-only infection were younger and had a high incidence of paroxysmal cough compared with patients with coinfection $(p<0.05)$. However, patients with coinfection had an increased demand for oxygen and showed more crackles in lungs $(p<0.05)$ (Table 4).

\section{DISCUSSION}

In recent years, an increasing incidence of pertussis has been reported in infants and young children (13). Several studies suggested that $B$. pertussis is a possible pathogen causing bronchiolitis in infants (8-10). Several investigators demonstrated that $B$. pertussis is a common pathogen of bronchiolitis $(14,15)$. A study conducted in Turkey identified $44(25.6 \%)$ of the 172 infants with B. pertussis hospitalized for acute bronchiolitis (15). Another study showed B. pertussis involvement in 12 of 142 (8.5\%) infants hospitalized for bronchiolitis in Finland (9). Yet other studies identified that $B$. pertussis was an uncommon pathogen in bronchiolitis (16). Pedro A. Piedra and his colleges found only four of 2,027 children admitted to the hospital as B. pertussis positive using PCR in the USA (10). Similarly, Walsh et al. found B. Pertussis infection in three of 488 patients $(0.6 \%)$ in the emergency department (17). The present study found that $7.1 \%$ of infants and young children hospitalized with acute bronchiolitis had a positive $B$. pertussis test, which demonstrated that $B$. pertussis was a common pathogen in bronchiolitis. The variation in the prevalence of $B$. pertussis in children hospitalized with bronchiolitis among the studies might be due to the difference in climates, recruit criteria, and vaccination. According to the finding of this study, the peak incidence of $B$. pertussis infection was from May to July, with a total of $33(50.15 \%)$ patients, which has not been reported before.

Studies reported that $B$. pertussis coinfection with other respiratory viruses was common in children hospitalized for bronchiolitis; the incidence rate was $36-67 \%(9,14,15,18)$. However, the present study reported that 45 (57.7\%) patients with $B$. pertussis were coinfected with other respiratory viruses, which was in agreement with previous studies. Some studies $(6,15,19)$ suggested that coinfection with RSV was the most common in young children hospitalized for bronchiolitis with $B$. pertussis infection. However, in the present study, the most common coinfection respiratory viruses in children with $B$. pertussis hospitalized for bronchiolitis were HRV (35.9\%), followed by MP (11.4\%), and $\mathrm{HBoV}(7.7 \%)$; these differences in coinfection might be due to the heterogeneity of social demography and differences in study methods and periods.

Symptom duration before admission and hospital stay were more common in $B$. pertussis-only infection than in RSV-only infection $(p<0.05)$. It indicated that patients with B. pertussisassociated bronchiolitis often presented a longer course, which was consistent with the clinical symptoms of $B$. pertussis infection (20) and could help distinguish B. pertussis-associated acute bronchiolitis from other acute bronchiolitis etiologies. The present study compared B. pertussis-only infection with RSV-only infection in children with bronchiolitis. Children with $B$. pertussis-only infection requiring supplement oxygen 
TABLE 2 | Select characteristics of hospitalized children with B. pertussis-only infection compared with RSV-only, MP-only, and other pathogens infection ( $n=1,092)$.

\begin{tabular}{|c|c|c|c|c|c|}
\hline & $\begin{array}{l}\text { B. pertussis-only } \\
\text { infection }(n=33)\end{array}$ & $\begin{array}{l}\text { RSV-only infection } \\
(n=438)\end{array}$ & $\begin{array}{l}\text { MP-only infection } \\
\quad(n=87)\end{array}$ & $\begin{array}{c}\text { Other pathogens } \\
\text { infection }(n=534)\end{array}$ & $p$-value \\
\hline Gender (male/female) & $21 / 12$ & $321 / 117$ & $54 / 33$ & $374 / 160$ & 0.148 \\
\hline \multicolumn{6}{|l|}{ Age group } \\
\hline$\leq 3$ months & $17(51.5 \%)$ & $300(68.5 \%)^{\star \star}$ & $30(34.5 \%)$ & $152(28.5 \%)^{\star}$ & $<0.001$ \\
\hline $12-24$ months & $2(6.1 \%)$ & $21(4.8 \%)$ & $27(31.0 \%)^{\star}$ & $138(25.8 \%)$ & $<0.001$ \\
\hline Duration of symptoms before admission (days) & $14.0(7.0,15.0)$ & $5.0(4.0,8.0)^{\star \star}$ & $9.0(5.0,17.0)$ & $10.0(5.0,15.0)$ & 0.001 \\
\hline \multicolumn{6}{|l|}{ Duration of symptoms before admission group } \\
\hline$<7 \mathrm{~d} n(\%)$ & $3(9.1 \%)$ & $285(65.1 \%)^{*}$ & $30(34.5 \%)^{\star}$ & $200(37.5 \%)^{*}$ & $<0.001$ \\
\hline $7-14 d n(\%)$ & $12(36.4 \%)$ & $72(16.4 \%)^{\star}$ & $30(34.5 \%)^{\star \star}$ & $139(26.0 \%)$ & $<0.001$ \\
\hline Dyspnea & $2(6.1 \%)$ & $18(4.1 \%)$ & $0(0.0 \%)^{\star *}$ & $54(10.1 \%)$ & $<0.001$ \\
\hline Rhinorrhea & $15(45.5 \%)$ & $195(44.5 \%)$ & $21(24.1 \%)^{\star \star}$ & $218(40.8 \%)$ & 0.005 \\
\hline Vomiting & $12(36.4 \%)$ & $78(17.8 \%)^{\star \star}$ & $9(10.3 \%)^{\star}$ & $117(21.9 \%)$ & 0.005 \\
\hline Cyanosis $n(\%)$ & $4(12.1 \%)$ & $12(2.7 \%)^{\star}$ & $0(0.0 \%)^{*}$ & $36(6.7 \%)$ & $<0.001$ \\
\hline $\mathrm{O}_{2}$ requirement $[n(\%)]$ & $2(6.1 \%)$ & $153(34.9 \%)^{*}$ & $12(13.8 \%)$ & $100(18.7 \%)$ & $<0.001$ \\
\hline Crackles n (\%) & $14(42.4 \%)$ & $291(66.4 \%)^{\star}$ & $54(62.1 \%)$ & $292(54.7 \%)$ & $<0.001$ \\
\hline \multicolumn{6}{|l|}{ Laboratory findings } \\
\hline Leukocyte count (× 109/L) & $16.8 \pm 6.7$ & $9.0 \pm 4.0^{*}$ & $9.5 \pm 3.7^{\star}$ & $11.3 \pm 5.9^{*}$ & $<0.001$ \\
\hline Leukocyte count $>15 \times 10^{9}$ & $19(57.6 \%)$ & $33(7.5 \%)^{\star}$ & $6(6.9 \%)^{\star}$ & $106(19.9 \%)^{\star}$ & $<0.001$ \\
\hline Lymphocyte count (\%) & $42.5 \pm 28.6$ & $60.8 \pm 13.4^{\star}$ & $48.2 \pm 18.4$ & $53.3 \pm 24.4$ & 0.006 \\
\hline Neutrophil count (\%) & $21.4 \pm 21.7$ & $32.9 \pm 44.1$ & $44.4 \pm 18.6^{\star \star}$ & $40.0 \pm 21.0$ & $<0.001$ \\
\hline
\end{tabular}

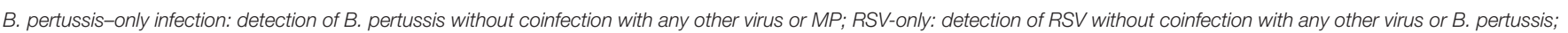

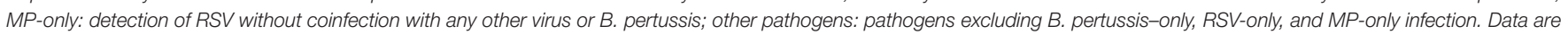

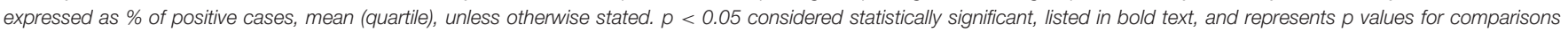

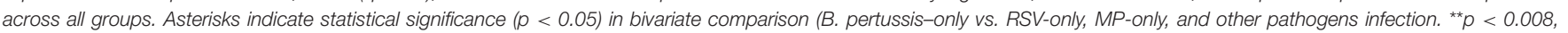
$\left.{ }^{\star} p<0.05\right)$.

were fewer than children with RSV-only infection, indicating that the former had a milder clinical presentation compared with the latter. This study also compared B. pertussis-only infection with MP-only infection and infections with other pathogens in children and revealed similar severity among these pathogens. This is a novel report explaining such associations. Several other studies (9, 14, 15, 18, 20-22) assessed the influence of $B$. pertussis on acute bronchiolitis, but they could not exclude the possibility of other respiratory pathogens contributing to the illness. In the present study, the leukocyte count was higher in patients with B. pertussisonly bronchiolitis infection than that in patients with RSVonly infection, MP-only infection, and infections with other pathogens ( $p<0.008$ for all comparisons), which could also help distinguish $B$. pertussis-associated acute bronchiolitis from other acute bronchiolitis etiologies. One study showed that the leukocyte count $>60 \times 10^{9} / \mathrm{L}$ was associated with death in children with $B$. pertussis infection (23). Another study demonstrated that the leukocyte count $>100 \times 10^{9} / \mathrm{L}$ was an independent risk factor of death in children with pertussis (24). However, no patent died of $B$. pertussis infection in the present study, which might be because the vast majority of infants and young children with mild-to-moderate bronchiolitis were considered, and severe bronchiolitis in the PICU setting was ignored.

Pertussis is a vaccine-preventable respiratory disease. $B$. pertussis could affect all individuals, but the highest morbidity and mortality rates were among newborns and unvaccinated or incompletely vaccinated young infants $(21,25,26)$. In the present study, the morbidity of $B$. pertussis was common $(31 / 78,39.7 \%)$ in infants with bronchiolitis who had been unvaccinated (infants $\leq 3$ months). The unvaccinated infants were associated with a longer hospital stay and more likely to require supplemental oxygen. Studies suggested that early identification and treatment of $B$. pertussis could shorten the duration of paroxysmal cough $(27,28)$, 
TABLE 3 | Clinical characteristics and results of laboratory examination among the different age groups with B. pertussis infection.

\begin{tabular}{|c|c|c|c|c|}
\hline & $\leq 3$ months $(n=31)$ & 4-11 months $(n=36)$ & $\geq 12$ months $(n=11)$ & $p$-value \\
\hline \multicolumn{5}{|l|}{ Clinical characteristics } \\
\hline Hospital stay (day) & $12.5 \pm 6.69$ & $9.91 \pm 3.41$ & $9 \pm 1.56$ & 0.049 \\
\hline Requirement for supplemental oxygen $n(\%)$ & $10(32.3 \%)$ & $2(5.6 \%)$ & $1(9.1 \%)$ & 0.011 \\
\hline Paroxysmal cough $n(\%)$ & $30(96.8 \%)$ & $33(91.7 \%)$ & $9(81.8 \%)$ & 0.237 \\
\hline Whoops $n(\%)$ & $3(9.7 \%)$ & $9(25.0 \%)$ & $0(0.0 \%)$ & 0.076 \\
\hline Low oxygen saturation $n(\%)^{a}$ & $3(9.7 \%)$ & $2(5.6 \%)$ & $1(9.1 \%)$ & 0.728 \\
\hline Crackles $n(\%)$ & $18(58.1 \%)$ & $27(75.0 \%)$ & $4(36.5 \%)$ & 0.051 \\
\hline \multicolumn{5}{|l|}{ Laboratory results } \\
\hline Leukocyte count (× 109/L) & $15.83 \pm 6.58$ & $17.72 \pm 8.39$ & $14.25 \pm 5.52$ & 0.356 \\
\hline Lymphocyte (\%) & $38.57 \pm 28.89$ & $51.42 \pm 26.63$ & $35.77 \pm 25.29$ & 0.102 \\
\hline
\end{tabular}

TABLE 4 | Comparison between B. pertussis-only infection and coinfection.

\begin{tabular}{|c|c|c|c|}
\hline & B. pertussis-only infection $(n=33)$ & B. pertussis and virus coinfection $(n=45)$ & $p$-value \\
\hline Gender (male) $n(\%)$ & $21(63.6 \%)$ & $25(55.6 \%)$ & 0.473 \\
\hline Vaccination & $22(66.7 \%)$ & $27(60.0 \%)$ & 0.547 \\
\hline Oxygen $n(\%)$ & $2(6.1 \%)$ & $11(24.4 \%)$ & 0.031 \\
\hline Paroxysmal cough $n(\%)$ & $33(100 \%)$ & 39 (86.7\%) & 0.036 \\
\hline Cyanosis n (\%) & $4(12.1 \%)$ & $6(13.3 \%)$ & 0.874 \\
\hline Low oxygen saturation $n(\%)^{a}$ & $2(6.1 \%)$ & $4(8.9 \%)$ & 0.643 \\
\hline Crackles n (\%) & $14(42.4 \%)$ & $35(77.8 \%)$ & 0.001 \\
\hline
\end{tabular}

B. pertussis-only infection: detection of B. pertussis without coinfection with any other virus or MP.

a Low oxygen saturation is less than $92 \%$.

and antibiotics against pertussis could limit the severity of disease if started in the catarrhal phase $(27,29)$. In addition, several systematic reviews confirmed the safety and effectiveness of maternal pertussis vaccination during pregnancy (30-32). Therefore, it is important to early recognize and initiate treatment.

This study had potential limitations. It enrolled only inpatients hospitalized with $B$. pertussis infection, but more patients with $B$. pertussis infection were treated in the outpatient department. Therefore, patients with more severe symptoms might have been overrepresented, and the prevalence of $B$. pertussis in children with bronchiolitis-associated hospitalization might be affected.

In summary, $B$. pertussis is one of the pathogens in children with bronchiolitis, and coinfection of $B$. pertussis with other viruses is common in bronchiolitis. $B$. pertussis should be considered when patients hospitalized with bronchiolitis present a longer course and have an elevated leukocyte count. Patients with B. pertussis-associated bronchiolitis present a milder clinical presentation.

\section{DATA AVAILABILITY STATEMENT}

The raw data supporting the conclusions of this article will be made available by the authors, without undue reservation.

\section{ETHICS STATEMENT}

The studies involving human participants were reviewed and approved by Ethics committees of Children's Hospital Soochow University (Approval No.: 2016026). Written informed consent to participate in this study was provided by the participants' legal guardian/next of kin.

\section{AUTHOR CONTRIBUTIONS}

WJ and SC wrote the main manuscript text. $\mathrm{CH}$ and $\mathrm{YW}$ designed the study and revised the manuscript. ZC and MW carried out the initial analyses. XS and JX performed the microbiological detection. $\mathrm{AL}$ and $\mathrm{QX}$ performed the data collection. All authors read and approved the final manuscript. 


\section{REFERENCES}

1. Bordet J, Gengou O. Le microbe de la coqueluche. Ann. I'Institut Pasteur. (1906) 20:731-741.

2. World Health Organization. Immunization Vaccines and Biologicals: Pertussis 2018 (2019). Available online at: https://www.who.int/immunization/ monitoring_surveillance/burden/vpd/surveillance_type/passive/pertussis/ en/.

3. Yeung K, Duclos P, Nelson E, Hutubessy R, Hutubessy RCW. An update of the global burden of pertussis in children younger than 5 years: a modelling study. Lancet Infect Dis. (2017) 17:974-80. doi: 10.1016/S1473-3099(17)30390-0

4. Carroll KN, Gebretsadik T, Griffin MR, Wu P, Dupont WD, Mitchel EF, et al. Increasing burden and risk factors for bronchiolitis-related medical visits in infants enrolled in a state health care insurance plan. Pediatrics. (2008) 122:58-64. doi: 10.1542/peds.2007-2087

5. Hasegawa K, Tsugawa Y, Brown D, Mansbach JM, Camargo CA. Trends in bronchiolitis hospitalizations in the United States, 2000-2009. Pediatrics. (2013) 132:28-36. doi: 10.1542/peds.2012-3877

6. Robledo-Aceves M, Moreno-Peregrina M, Velarde-Rivera F, AscencioEsparza E, Preciado-Figueroa FM, Caniza MA, et al. Risk factors for severe bronchiolitis caused by respiratory virus infections among Mexican children in an emergency department. Medicine. (2018) 97:e0057. doi: 10.1097/MD.0000000000010057

7. Cui D, Feng L, Chen Y, Lai S, Zhang Z, Yu F, et al. Clinical and epidemiologic characteristics of hospitalized patients with laboratoryconfirmed respiratory syncytial virus infection in eastern China between 2009 and 2013: a retrospective study. PLoS ONE. (2016) 11:e0165437. doi: 10.1371/journal.pone.0165437

8. Heininger U, Burckhardt MA. Bordetella pertussis and concomitant viral respiratory tract infections are rare in children with cough illness. Pediatr Infect Dis J. (2011) 30:640-4. doi: 10.1097/INF.0b013e3182152d28

9. Nuolivirta K, Koponen P, He Q, Halkosalo A, Korppi M, Vesikari $\mathrm{T}$, et al. Bordetella pertussis infection is common in nonvaccinated infants admitted for bronchiolitis. Pediatr Infect Dis J. (2010) 29:1013-5. doi: 10.1097/INF.0b013e3181f537c6

10. Piedra PA, Mansbach JM, Jewell AM, Thakar SD, Camargo CA. Bordetella pertussis is an uncommon pathogen in children hospitalized with bronchiolitis during the winter season. Pediatr Infect Dis J. (2015) 34:566-70. doi: 10.1097/INF.0000000000000596

11. Ralston SL, Lieberthal AS, Meissner HC, Alverson BK, Baley JE, Gadomski $\mathrm{AM}$, et al. Clinical practice guideline: the diagnosis, management, and prevention of bronchiolitis. Pediatrics. (2014) 134:e1474-502. doi: 10.1542/peds.2014-2742

12. Cherry JD, Tan T, von König Carl-Heinz W, Forsyth KD, Usa T, David G, et al. Clinical definitions of pertussis: summary of a global pertussis initiative roundtable meeting, February 2011. Clin Infect Dis. (2012) 54:1756-64. doi: $10.1093 / \mathrm{cid} / \mathrm{cis} 302$

13. Wood N, Mcintyre P. Pertussis: review of epidemiology, diagnosis, management and prevention. Paediatr Respir Rev. (2008) 9:201-11; quiz 211-2. doi: 10.1016/j.prrv.2008.05.010

14. Raya BA, Bamberger E, Kassis I, Kugelman A, Srugo I, Miron, et al. Bordetella pertussis infection attenuates clinical course of acute bronchiolitis. Pediatr Infect Dis J. (2013) 32:619-21. doi: 10.1097/INF.0b013e3182877973

15. Gökçe S, Kurugöl Z, Söhret Aydemir S, Çiçek C, Aslan A, Koturoglu G. Bordetella pertussis infection in hospitalized infants with acute bronchiolitis. Indian J Pediatr. (2018) 85:189-93. doi: 10.1007/s12098-017-2480-4

16. Efendiyeva E, Kara TT, Erat T, Yahi A, Ifti E. The incidence and clinical effects of Bordetella pertussis in children hospitalized with acute bronchiolitis. Turk J Pediatr. (2020) 62:726-33. doi: 10.24953/turkjped.2020.05.003

17. Walsh P, Overmeyer C, Kimmel L, Feola M, Adelson ME. Prevalence of Bordetella pertussis and Bordetella parapertussis in Samples Submitted for RSV screening. West J Emerg Med. (2008) 9:135-40.

18. Greenberg D, Bamberger E, Ben-Shimol S, Gershtein R, Srugo, I. Pertussis is under diagnosed in infants hospitalized withlower respiratory tract infection in the pediatric intensive care unit. Med Sci Monit. (2007) 13:CR475-480.
19. Sun H, Ji Y, Ji W, Hao C, Yan Y, Chen Z. Impact of RSV coinfection on human bocavirus in children with acute respiratory infections. J Trop Pediatr. (2019) 65:342-51. doi: 10.1093/tropej/fmy057

20. Melvin JA, Scheller EV, Miller JF, Cotter PA. Bordetella pertussis pathogenesis: current and future challenges. Nat Rev Microbiol. (2014) 12:274-88. doi: $10.1038 / \mathrm{nrmicro3235}$

21. Somerville RL, Grant CC, Grimwood K, Murdoch D, Graham D, Jackson $\mathrm{P}$, et al. Infants hospitalised with pertussis: estimating the true disease burden. J Paediatr Child Health. (2007) 43:617-22. doi: 10.1111/j.1440-1754.2007.01154.x

22. Jiang $\mathrm{W}, \mathrm{Wu} \mathrm{M}$, Chen $\mathrm{S}, \mathrm{Li} \mathrm{A}, \mathrm{Xu} \mathrm{J}$, Wang $\mathrm{Y}$, et al. Share virus coinfection is a predictor of radiologically confirmed pneumonia in children with bordetella pertussis infection. Infect Dis Ther. (2020) 10:335-46. doi: 10.1007/s40121-020-00376-5

23. Paddock C, Sanden G, Cherry J, Langston C, Tatti K, Wu KH, et al. Pathology and pathogenesis of fatal bordetella pertussis infection in infants. Clin Infect Dis. (2008) 47:328-38. doi: 10.1086/589753

24. Pierce C, Klein N, Peters M. Is leukocytosis a predictor of mortality in severe pertussis infection? Intens Care Med. (2014) 26:1512-4. doi: $10.1007 /$ s001340000587

25. Tanaka M, Vitek CR, Brain Pascual F, Bisgard KM, Tate JE, Murphy TV. Trends in pertussis among infants in the United States, 1980-1999. JAMA. (2003) 290:2968-75. doi: 10.1001/jama.290.22.2968

26. Masseria C, Martin CK, Krishnarajah G, Becker LK, Buikema A, Tan TQ, et al. Incidence and burden of pertussis among infants less than 1 year of age. Pediatr Infect Dis J. (2017) 36:e54-61. doi: 10.1097/INF.0000000000001440

27. Carlsson R, Segebaden KV, Bergstrom J, Kling A, Nilsson, L. Surveillance of infant pertussis in Sweden 1998-2012: severity of disease in relation to the national vaccination programme. Euro Surveill. (2015) 20:21032. doi: 10.2807/1560-7917.ES2015.20.6.21032

28. Tiwari TS BA, Clark TA. First pertussis vaccine dose and prevention of infant mortality. Pediatrics. (2015) 135:990-9. doi: 10.1542/peds.2014-2291

29. Bergquist SO, Brenander S, Dahnsjö H, Sundelöf B. Erythromycin in the treatment of pertussis- a study of bacteriologic and clinical effects. Pediatr Infect Dis J. (1987) 6:458-61. doi: 10.1097/00006454-19870500000009

30. Switzer C, D'Heilly C, Macina D. Immunological and clinical benefits of maternal immunization against pertussis: a systematic review. Infect Dis Ther. (2019) 8:499-541. doi: 10.1007/s40121-019-00264-7

31. Heilly CD, Switzer C, Macina D. Safety of maternal immunization against pertussis: a systematic review. Infect Dis Ther. (2019) 8:543-68. doi: 10.1007/s40121-019-00265-6

32. Ashish A, Sanjeev S, Kolhapure S, Kandeil W, Pai R, Singhal T. Neonatal pertussis, an under-recognized health burden and rationale for maternal immunization: a systematic review of south and South-East Asian Countries. Infect Dis Ther. (2019) 8:139-53. doi: 10.1007/s40121-019-0245-2

Conflict of Interest: The authors declare that the research was conducted in the absence of any commercial or financial relationships that could be construed as a potential conflict of interest.

Publisher's Note: All claims expressed in this article are solely those of the authors and do not necessarily represent those of their affiliated organizations, or those of the publisher, the editors and the reviewers. Any product that may be evaluated in this article, or claim that may be made by its manufacturer, is not guaranteed or endorsed by the publisher.

Copyright (c) 2021 Chen, Wang, Li, Jiang, Xu, Wu, Chen, Hao, Shao and Xu. This is an open-access article distributed under the terms of the Creative Commons Attribution License (CC BY). The use, distribution or reproduction in other forums is permitted, provided the original author(s) and the copyright owner(s) are credited and that the original publication in this journal is cited, in accordance with accepted academic practice. No use, distribution or reproduction is permitted which does not comply with these terms. 